\title{
DEKOMPOSISI PRA A*-ALJABAR
}

\author{
RAHMIATI ABAS \\ Program Studi Matematika, \\ Fakultas Matematika dan Ilmu Pengetahuan Alam, Universitas Andalas Padang, \\ Kampus UNAND Limau Manis Padang, Indonesia \\ rahmiati.abas@gmail.com
}

\begin{abstract}
Abstrak. Suatu sistem matematika $\left(A, \wedge, \vee,(\cdot)^{\sim}\right)$ dinamakan Pra $\mathrm{A}^{*}$-Aljabar, bila anggota-anggotanya memenuhi sifat-sifat tertentu. Untuk selanjutnya, sistem $(A, \wedge, \vee$, $\left.(\cdot)^{\sim}\right)$ ditulis $A$ yang menyatakan Pra A*-Aljabar. Misal didefinisikan sebuah relasi " $\leq$ " pada Pra $\mathrm{A}^{*}$-Aljabar dengan $x \leq y$ jika dan hanya jika $y \wedge x=x \wedge y=x$. Selanjutnya misalkan terdapat suatu sistem matematika $\left(M_{x}, \wedge, \vee, *\right)$ dengan $M_{x}=\{s \in A \mid s \leq x\}$. Misalkan terdapat himpunan $B(A)=\left\{x \in A \mid x \vee x^{\sim}=1\right\}$ yang disebut senter (centre) dari $A$, dan didefinisikan $M_{a}^{\prime}=\{s \in B(A) \mid s \leq a\}$ dan $M_{a}^{\prime} \sim=\left\{t \in B(A) \mid t \leq a^{\sim}\right\}$. Pada tulisan ini dikaji sifat-sifat dari $M_{x}$, yaitu: suatu sistem $\left(M_{x}, \wedge, \vee, *\right)$ adalah Pra A*-Aljabar dengan unsur identitas $1, M_{x}=\{x \wedge s \mid s \in A\}, M_{x}=M_{y}$ jika dan hanya jika $x=y, M_{x} \cap M_{y}=M_{x \wedge y},\left(M_{x}\right)_{x \wedge y}=M_{x \wedge y}$, dan pemetaan $\alpha_{x}: A \rightarrow M_{x}$ adalah sebuah homomorfisma pada. Kemudian juga dikaji bahwa $A$ monomorfik dengan $M_{a} \times M_{a} \sim$ dan $B(A) \cong M_{a}^{\prime} \times M_{a}^{\prime} \sim$

Kata Kunci: Aljabar Boolean, Pra A*-Aljabar, Pra A*-Homomorfisma, Dekomposisi Pra A*-Aljabar
\end{abstract}

\section{Pendahuluan}

Gagasan Pra A*-Aljabar pertama kali diperkenalkan pada tahun 2000 oleh J. Venkateswara Rao. Pra A*-Aljabar merupakan suatu sistem matematika $(A, \wedge, \vee$, $\left.(\cdot)^{\sim}\right)$ dimana $A$ adalah himpunan tak kosong, $\wedge$ (meet) dan $\vee($ join) adalah operasioperasi biner, dan $(\cdot)^{\sim}$ (tilda) adalah operasi tunggal, yang anggota-anggotanya memenuhi sifat-sifat: $x^{\sim \sim}=x, x \wedge x=x, x \wedge y=y \wedge x,(x \wedge y)^{\sim}=x^{\sim} \vee y^{\sim}$, $x \wedge(y \wedge z)=(x \wedge y) \wedge z, x \wedge(y \vee z)=(x \wedge y) \vee(x \wedge z)$, dan $(x \wedge y)=x \wedge\left(x^{\sim} \vee y\right)$.

Misalkan didefinisikan sebuah relasi " $\leq$ " pada Pra A*-Aljabar dengan $s \leq x$ jika dan hanya jika $s \wedge x=x \wedge s=s$. Selanjtunya misalkan terdapat suatu sistem matematika $\left(M_{x}, \wedge, \vee, *\right)$ dengan $M_{x}=\{s \in A \mid s \leq x\}$.

Misalkan terdapat himpunan $B(A)=\left\{x \in A \mid x \vee x^{\sim}=1\right\}$ yang disebut senter (centre) dari $A$. Kemudian didefinisikan $M_{a}^{\prime}=\{s \in B(A) \mid s \leq a\}$ dan $M_{a}^{\prime}=\{t \in$ $\left.B(A) \mid t \leq a^{\sim}\right\}$.

Makalah ini merupakan tinjauan ulang dari rujukan pustaka [2]. Pada makalah ini penulis mengkaji kembali tentang Dekomposisi Pra A*-Aljabar.

\section{Pra $A^{*}$-Aljabar}

Pada tulisan, diberikan definisi dari Pra A*-Aljabar dan sifat-sifat dari Pra A*Aljabar. 
Definisi 2.1. [3] Misal A adalah himpunan tak kosong. Suatu sistem matematika $\left(A, \wedge, \vee,(\cdot)^{\sim}\right)$ dikatakan Pra $A^{*}$-Aljabar, dengan $\wedge$ (meet) dan $\vee$ (join) adalah operasi-operasi biner, dan $(\cdot)^{\sim}$ (tilda) adalah operasi tunggal, jika untuk setiap $x, y, z, \in A$, berlaku: (i) $x^{\sim \sim}=x$, (ii) $x \wedge x=x$, (iii) $x \wedge y=y \wedge x$, (iv) $(x \wedge y)^{\sim}=x^{\sim} \vee y^{\sim},(v) x \wedge(y \wedge z)=(x \wedge y) \wedge z,(v i) x \wedge(y \vee z)=(x \wedge y) \vee(x \wedge z)$, (vii) $(x \wedge y)=x \wedge\left(x^{\sim} \vee y\right)$.

Definisi 2.2. Misalkan sistem $\left(A, \wedge, \vee,(\cdot)^{\sim}\right)$ adalah Pra $A^{*}$-Aljabar dengan unsur identitas 1 dan 0 . Suatu sistem lain, namakan $A^{*}$ dinamakan dual dari $A$ jika:

(1) $\wedge$ diganti dengan $\vee$,

(2) $\vee$ diganti dengan $\wedge$,

(3) 0 diganti dengan 1 ,

(4) 1 diganti dengan 0 .

Contoh 2.3. Himpunan $\mathbf{W}=\{0,1,2\}$ adalah suatu Pra $A^{*}$-Aljabar dengan operasi $\vee, \wedge$, dan $(\cdot)^{\sim}$ yang didefinisikan seperti pada tabel berikut:

\begin{tabular}{|l|l|l|l|}
\hline$\wedge$ & 0 & 1 & 2 \\
\hline 0 & 0 & 0 & 2 \\
\hline 1 & 0 & 1 & 2 \\
\hline 2 & 2 & 2 & 2 \\
\hline
\end{tabular}

Tabel 1. Operasi Biner $\wedge$ pada $A$

\begin{tabular}{|c|c|c|c|}
\hline$\vee$ & 0 & 1 & 2 \\
\hline 0 & 0 & 1 & 2 \\
\hline 1 & 1 & 1 & 2 \\
\hline 2 & 2 & 2 & 2 \\
\hline
\end{tabular}

Tabel 2. Operasi Biner $\vee$ pada $A$

\begin{tabular}{|c|c|}
\hline$x$ & $x^{\sim}$ \\
\hline 0 & 1 \\
\hline 1 & 0 \\
\hline 2 & 2 \\
\hline
\end{tabular}

Tabel 3. Operasi $\sim$ pada $A$

Definisi 2.4. [3] Misalkan A adalah Pra $A^{*}$-Aljabar. Suatu unsur $x \in A$ disebut elemen sentral dari $A$ jika $x \vee x^{\sim}=1$. Selanjutnya, himpunan $\left\{x \in A \mid x \vee x^{\sim}=1\right\}$ 
disebut sebagai himpunan semua elemen sentral dari $A$, yang disebut senter (centre) dari $A$ dan dinotasikan dengan $B(A)$.

Teorema 2.5. [3] Misalkan $A$ adalah Pra $A^{*}$-Aljabar dengan unsur identitas 1, maka $B(A)$ adalah aljabar Boolean dengan operasi $\vee, \wedge,(\cdot)^{\sim}$.

Definisi 2.6. [3] Misalkan $A_{1}$ dan $A_{2}$ merupakan dua Pra $A^{*}$-Aljabar. Pemetaan $f: A_{1} \rightarrow A_{2}$ disebut Pra $A^{*}$-homomorfisma jika untuk setiap a, $b \in A_{1}$ berlaku:

(1) $f(a \wedge b)=f(a) \wedge f(b)$,

(2) $f(a \vee b)=f(a) \vee f(b)$,

(3) $f\left(a^{\sim}\right)=(f(a))^{\sim}$.

Jika homomorfisma $f: A_{1} \rightarrow A_{2}$ adalah pada, maka $f$ disebut epimorfisma. Jika homomorfisma $f: A_{1} \rightarrow A_{2}$ adalah satu - satu, maka $f$ disebut monomorfisma. Jika homomorfisma $f: A_{1} \rightarrow A_{2}$ adalah satu - satu dan pada, maka $f$ disebut isomorfisma, dan $A_{1}, A_{2}$ adalah isomorfik, yang dinotasikan dengan $A_{1} \cong A_{2}$.

Definisi 2.7. Misalkan A adalah Pra $A^{*}$-Aljabar. Didefinisikan sebuah relasi" $\leq "$ pada $A$ dengan $x \leq y$ jika dan hanya jika $y \wedge x=x \wedge y=x$, untuk setiap $x, y \in A$.

\section{Pra A*-Aljabar $M_{x}$}

Pada tulisan, akan dibahas sifat dari sistem $\left(M_{x}, \wedge, \vee, *\right)$ yang dinyatakan dalam beberapa teorema berikut.

Teorema 3.1. [2] Misalkan $A$ adalah sebuah Pra $A^{*}$-Aljabar dengan $x \in A$, dan $M_{x}=\{s \in A \mid s \wedge x=x \wedge s=s\}$. Maka $\left(M_{x}, \wedge, \vee, *\right)$ adalah Pra $A^{*}$-Aljabar dengan unsur identitas 1 , dimana $\wedge$ dan $\vee$ adalah operasi biner di $A$ yang dibatasi pada $M_{x}$.

Bukti. Misalkan $x \in A$ dan $M_{x}=\{s \in A \mid s \wedge x=x \wedge s=s\}$. Sebelumnya akan ditunjukkan bahwa jika $s \in M_{x}$, maka $s^{*} \in M_{x}$. Misalkan $s \in M_{x}$, berarti $s \in A$ dan $s \wedge x=x \wedge s=s$. Karena $s \in A$, maka $s^{*} \in A$. Akibatnya $s^{*} \wedge x=\left(x \wedge s^{\sim}\right) \wedge x=$ $\left(s^{\sim} \wedge x\right) \wedge x=s^{\sim} \wedge(x \wedge x)=s^{\sim} \wedge x=x \wedge s^{\sim}=s^{*}$, sehingga $s^{*} \in M_{x}$.

Selanjutnya akan ditunjukkan $\left(M_{x}, \wedge, \vee, *\right)$ adalah Pra $A^{*}$-Aljabar dengan unsur identitas 1. Berdasarkan Definisi 2.3.6, cukup ditunjukkan:

(1) Misalkan $s \in M_{x}$. Akan ditunjukkan $s^{* *}=s$.

Perhatikan bahwa: $s^{* *}=\left(s^{*}\right)^{*}=\left(x \wedge s^{\sim}\right)^{*}=\left(x \wedge\left(x \wedge s^{\sim}\right)^{\sim}\right)=\left(x \wedge\left(x^{\sim} \vee s\right)=\right.$ $x \wedge s=s$.

(2) Misalkan $s, t \in M_{x}$. Akan ditunjukkan $(s \wedge t)^{*}=s^{*} \vee t^{*}$.

Perhatikan bahwa: $(s \wedge t)^{*}=x \wedge(s \wedge t)^{\sim}=x \wedge\left(s^{\sim} \vee t^{\sim}\right)=\left(x \wedge s^{\sim}\right) \vee\left(x \wedge t^{\sim}\right)=$ $s^{*} \vee t^{*}$.

(3) Misalkan $s, t \in M_{x}$. Akan ditunjukkan $s \wedge t=s \wedge\left(s^{*} \vee t\right)$.

Perhatikan bahwa: $s \wedge\left(s^{*} \vee t\right)=s \wedge\left(\left(x \wedge s^{\sim}\right) \vee t\right)=\left(s \wedge\left(x \wedge s^{\sim}\right)\right) \vee(s \wedge t)=$ $\left(s \wedge x \wedge s^{\sim}\right) \vee(s \wedge t)=\left((s \wedge x) \wedge s^{\sim}\right) \vee(s \wedge t)=\left(s \wedge s^{\sim}\right) \vee(s \wedge t)=\left(s^{\sim} \wedge s\right) \vee(s \wedge t)=$ $(s \wedge t) \vee\left(s^{\sim} \wedge s\right)=\left(s^{\sim} \vee t\right) \wedge s=t \wedge s=s \wedge t$. 
Untuk sifat-sifat lainnya, selama anggota dari sifat-sifat tersebut berada di $A$ maka anggota dari sifat-sifat tersebut juga berada di $M_{x}$. Oleh karena itu, $\left(M_{x}, \wedge, \vee, *\right)$ adalah Pra $\mathrm{A}^{*}$-Aljabar.

Teorema 3.2. [2] Misalkan A sebuah Pra $A^{*}$-Aljabar, maka untuk setiap $x, y \in A$ berlaku:

(1) $M_{x}=\{x \wedge s \mid s \in A\}$,

(2) $M_{x}=M_{y}$ jika dan hanya jika $x=y$,

(3) $M_{x} \cap M_{y}=M_{x \wedge y}$,

(4) $\left(M_{x}\right)_{x \wedge y}=M_{x \wedge y}$.

Bukti. Misalkan $A$ sebuah Pra A*-Aljabar dan misalkan $x, y \in A$.

(1) Jelas dari definisi.

(2) $(\Rightarrow)$ Misalkan $M_{x}=M_{y}$, berarti $s \in M_{x}, s \in M_{y}$. Akan ditunjukkan $x=y$. Andaikan $x \neq y$, maka $s \wedge x \neq s \wedge y$. Hal ini kontradiksi dengan $M_{x}=M_{y}$. Jadi haruslah $x=y$.

$(\Leftarrow)$ Misalkan $x=y$. Akan ditunjukkan $M_{x}=M_{y}$, yaitu dengan menunjukkan (i) $M_{x} \subset M_{y}$, (ii) $M_{x} \supset M_{y}$.

(i) Misalkan $z \in M_{x}$, maka berlaku $z \in A$ dan $z \wedge x=x \wedge z=z$. Karena $x=y$, maka $z \wedge y=y \wedge z=z$. Jadi $z \in M_{y}$. Oleh karena itu $M_{x} \subset M_{y}$.

(ii) Misalkan $z \in M_{y}$, maka berlaku $z \in A$ dan $z \wedge y=y \wedge z=z$. Karena $x=y$, maka $z \wedge x=x \wedge z=z$. Jadi $z \in M_{x}$. Oleh karena itu $M_{x} \supset M_{y}$.

Dari (i) dan (ii), maka $M_{x}=M_{y}$.

(3) Akan ditunjukkan $M_{x} \cap M_{y}=M_{x \wedge y}$, yaitu dengan menunjukkan (i) $M_{x} \cap M_{y} \subset$ $M_{x \wedge y}$, (ii) $M_{x} \cap M_{y} \supset M_{x \wedge y}$.

(i) Misalkan $s \in M_{x} \cap M_{y}$, maka $s \in M_{x}$ dan $s \in M_{y}$.

Dari $s \in M_{x}$ diperoleh $s \in A$ dan $s \wedge x=x \wedge s=s$. Sedangkan, dari $s \in M_{y}$ diperoleh $s \in A$ dan $s \wedge y=y \wedge s=s$.

Perhatikan bahwa: $(x \wedge y) \wedge s=x \wedge(y \wedge s)=x \wedge s=s$.

Jadi diperoleh $s \in A$ dan $(x \wedge y) \wedge s=s \wedge(x \wedge y)$, sehingga $s \in M_{x \wedge y}$. Oleh karena itu, $M_{x} \cap M_{y} \subset M_{x \wedge y}$.

(ii) Misalkan $s \in M_{x \wedge y}$ berarti $s \in A$ dan $(x \wedge y) \wedge s=s \wedge(x \wedge y)=s$.

Perhatikan bahwa: $x \wedge s=x \wedge((x \wedge y) \wedge s)=(x \wedge x) \wedge y \wedge s=(x \wedge y) \wedge s=s$.

Jadi $s \in A$ dan $x \wedge s=s \wedge x=s$, sehingga $s \in M_{x}$.

Dan, perhatikan bahwa: $y \wedge s=y \wedge((x \wedge y) \wedge s)=(y \wedge y) \wedge x \wedge s=y \wedge x \wedge s=$ $(x \wedge y) \wedge s=s$.

Jadi $s \in A$ dan $y \wedge s=s \wedge y=s$, sehingga $s \in M_{y}$. Karena $s \in M_{x}$ dan $s \in M_{y}$, maka $M_{x} \cap M_{y} \supset M_{x \wedge y}$.

Dari (i) dan (ii), maka $M_{x} \cap M_{y}=M_{x \wedge y}$.

(4) Misalkan $t \in M_{x}$, maka $\left(M_{x}\right)_{x \wedge y}=\left\{(x \wedge y) \wedge t \mid t \in M_{x}\right\}=\{(x \wedge y) \wedge(x \wedge s) \mid s \in$ $A\}=\{(x \wedge y) \wedge s \mid s \in A\}=M_{x \wedge y}$.

Teorema 3.3. [2] Misalkan $A$ adalah sebuah Pra $A^{*}$-Aljabar dengan unsur identitas 1 dan $x \in A$, maka pemetaan $\alpha_{x}: A \rightarrow M_{x}$ yang didefinisikan sebagai $\alpha_{x}(s)=x \wedge s$ untuk setiap $s \in A$, adalah sebuah homomorfisma pada. 
Bukti. Misalkan $s, t \in A$. Sebelumnya, akan ditunjukkan $\alpha_{x}$ adalah sebuah homomorfisma.

Perhatikan bahwa

(1) $\alpha_{x}(s \wedge t)=x \wedge(s \wedge t)=(x \wedge s) \wedge(x \wedge t)=\alpha_{x}(s) \wedge \alpha_{x}(t)$.

(2) $\alpha_{x}(s \vee t)=x \wedge(s \vee t)=(x \wedge s) \vee(x \wedge t)=\alpha_{x}(s) \vee \alpha_{x}(t)$.

(3) $\alpha_{x}\left(s^{\sim}\right)=x \wedge s^{\sim}=x \wedge\left(x^{\sim} \vee s^{\sim}\right)=x \wedge(x \wedge s)^{\sim}=(x \wedge t)^{*}=\left(\alpha_{x}\left(s^{\sim}\right)\right)^{*}$.

Selanjutnya ambil $s \in M_{x}$, akan dicari $s \in A$ sehingga $s=\alpha_{x}(s)$. Karena $s \wedge x=s$ dan diketahui $\alpha_{x}(s)=s \wedge x$, maka terdapat $s \in A$ sedemikian sehingga $\alpha_{x}(s)=s$. Oleh karena itu, $\alpha_{x}$ adalah homomorfisma pada.

\section{Dekomposisi dari $A$}

Pada tulisan, akan dibahas kaitan antara suatu Pra $\mathrm{A}^{*}$-Aljabar $A$ dengan dekomposisi Pra $\mathrm{A}^{*}$-Aljabar $M_{a} \times M_{a} \sim$ dan himpunan semua elemen sentral dari $A, B(A)$ dengan dekomposisi Pra $\mathrm{A}^{*}$-Aljabar $M_{a}^{\prime} \times M_{a}^{\prime} \sim$. Pertama-tama, akan dibuktikan lema berikut yang digunakan untuk membuktikan hasil utama pada bagian ini.

Lema 4.1. [2] Misalkan $A$ adalah Pra $A^{*}$-Aljabar dengan unsur identitas 1, dan $B(A)$ adalah himpunan semua elemen sentral dari $A$. Misal $a \in B(A)$, dan $x, y \in A$. Maka $a \wedge x=a \wedge y$ dan $a^{\sim} \wedge x=a^{\sim} \wedge y \Leftrightarrow x=y$.

Bukti. Misalkan $a \in B(A)$ dan $x, y \in A$.

$(\Rightarrow)$ Misalkan $a \wedge x=a \wedge y$ dan $a^{\sim} \wedge x=a^{\sim} \wedge y$. Akan ditunjukkan $x=y$.

Perhatikan bahwa: $x=1 \wedge x=\left(a \vee a^{\sim}\right) \wedge x=(a \wedge x) \vee\left(a^{\sim} \wedge x\right)=(a \wedge y) \vee\left(a^{\sim} \wedge y\right)=$ $\left(a \vee a^{\sim}\right) \wedge y=1 \wedge y=y$.

$(\Leftarrow)$ Misalkan $x=y$. Akan ditunjukkan $a \wedge x=a \wedge y$ dan $a^{\sim} \wedge x=a^{\sim} \wedge y$. Karena $x=y$, maka $a \wedge x=a \wedge y$ dan $a^{\sim} \wedge x=a^{\sim} \wedge y$.

Definisi 4.2. Misalkan $A_{1}, A_{2}$ adalah dua Pra $A^{*}$-Aljabar dengan $A_{1} \times A_{2}=$ $\left\{\left(a_{1}, a_{2}\right) \mid a_{1} \in A_{1}, a_{2} \in A_{2}\right\}$. Untuk setiap $a_{1}, a_{3} \in A_{1}, a_{2}, a_{4} \in A_{2}$, berlaku sifatsifat berikut:

(1) $\left(a_{1}, a_{2}\right) \wedge\left(a_{3}, a_{4}\right)=\left(a_{1} \wedge a_{3}, a_{2} \wedge a_{4}\right)$,

(2) $\left(a_{1}, a_{2}\right) \vee\left(a_{3}, a_{4}\right)=\left(a_{1} \vee a_{3}, a_{2} \vee a_{4}\right)$,

(3) $\left(a_{1}, a_{2}\right)^{\sim}=\left(a_{1}^{\sim}, a_{2}^{\sim}\right)$.

Teorema 4.3. [2] Misalkan A adalah sebuah Pra $A^{*}$-Aljabar dengan unsur identitas 1 dan $a \in B(A)$, maka $A$ monomorfik dengan $M_{a} \times M_{a} \sim$.

Bukti. Misalkan $a \in B(A)$ dan $x, y \in A$. Didefinisikan $\alpha: A \rightarrow M_{a} \times M_{a} \sim$ dengan $\alpha(x)=\left(\alpha_{a}(x), \alpha_{a} \sim(x)\right), \forall x \in A$.

Pertama-tama, akan ditunjukkan $\alpha$ terdefinisi dengan baik. Ambil $x, y \in A$ dengan $x=y$. Akan ditunjukkan $\alpha(x)=\alpha(y)$. Karena $x=y$, maka berdasarkan Lema 1 berlaku $a \wedge x=a \wedge y$ dan $a^{\sim} \wedge x=a^{\sim} \wedge y$. Akibatnya $\alpha(x)=\left(\alpha_{a}(x), \alpha_{a \sim}(x)\right)=$ $\left(a \wedge x, a^{\sim} \wedge x\right)=\left(a \wedge y, a^{\sim} \wedge y=\left(\alpha_{a}(y), \alpha_{a \sim}(y)\right)=\alpha(y)\right.$.

Kemudian, akan ditunjukkan bahwa $\alpha$ adalah homomorfisma.

Perhatikan bahwa: 
(1) $\alpha(x \wedge y)=\left(\alpha_{a}(x \wedge y), \alpha_{a} \sim(x \wedge y)\right)=\left(\alpha_{a}(x) \wedge \alpha_{a}(y), \alpha_{a} \sim(x) \wedge \alpha_{a^{\sim}}(y)\right)=$ $\left(\alpha_{a}(x) \wedge \alpha_{a} \sim(x), \alpha_{a}(y) \wedge \alpha_{a \sim}(y)\right)=\alpha(x) \wedge \alpha(y)$.

(2) $\alpha(x \vee y)=\left(\alpha_{a}(x \vee y), \alpha_{a \sim}(x \vee y)\right)=\left(\alpha_{a}(x) \vee \alpha_{a}(y), \alpha_{a \sim}(x) \vee \alpha_{a} \sim(y)\right)=$ $\left(\alpha_{a}(x) \vee \alpha_{a^{\sim}}(x), \alpha_{a}(y) \vee \alpha_{a} \sim(y)\right)=\alpha(x) \vee \alpha(y)$.

(3) $\alpha\left(x^{\sim}\right)=\left(\alpha_{a}\left(x^{\sim}\right), \alpha_{a \sim}\left(x^{\sim}\right)\right)=\left(\left(\alpha_{a}(x)\right)^{\sim},\left(\alpha_{a \sim}(x)\right)^{\sim}\right)=\left(\left(\alpha_{a}(x), \alpha_{a \sim}(x)\right)^{\sim}\right)=$ $(\alpha(x))^{\sim}$.

Selanjutnya, akan ditunjukkan $\alpha$ adalah satu-satu. Misal $\alpha(x)=\alpha(y)$, maka $a \wedge x=a \wedge y$ dan $a^{\sim} \wedge x=a^{\sim} \wedge y$. Sehingga berdasarkan Lema 1, diperoleh $x=y$. Karena $\alpha$ homomorfisma dan satu-satu, maka $\alpha$ adalah monomorfisma, sehingga $\alpha$ monomorfik dengan $M_{a} \times M_{a^{\sim}}$.

Teorema 4.4. [2] Misalkan A adalah sebuah Pra $A^{*}$-Aljabar dengan unsur identitas 1 dan $B(A)$ adalah himpunan semua elemen sentral dari $A$. Misal $a \in B(A)$, dan notasikan $M_{a}^{\prime}=\{s \in B(A) \mid s \leq a\}$ dan $M_{a^{\sim}}^{\prime}=\left\{t \in B(A) \mid t \leq a^{\sim}\right\}$. Maka $B(A) \cong M_{a}^{\prime} \times M_{a \sim}^{\prime}$.

Bukti. Misalkan $a \in B(A)$. Didefinisikan $\beta: B(A) \rightarrow M_{a}^{\prime} \times M_{a^{\sim}}^{\prime}$ dengan $\beta(x)=$ $\left(\beta_{a}(x), \beta_{a \sim}(x)\right), \forall x \in B(A)$.

Karena pendefinisian $\beta$ analog dengan $\alpha$ pada Teorema 5 , maka $\beta$ adalah monomorfisma.

Selanjutnya, akan ditunjukkan $\beta$ adalah suatu epimorfisma. Ambil $(x, y) \in M_{a}^{\prime} \times$ $M_{a}^{\prime} \sim$ sebarang. Berarti $x \in B(A)$ dan $x \leq a$, dan $y \in B(A)$ dan $y \leq a^{\sim}$. Karena $x \leq a$ diperoleh $a \wedge x=x$, selanjutnya karena $y \leq a^{\sim}$ diperoleh $a^{\sim} \wedge y=y$. Akibatnya, $a^{\sim} \wedge x=a^{\sim} \wedge a \wedge x=1 \wedge x=0$ dan $a \wedge y=a \wedge a^{\sim} \wedge y=1 \wedge y=0$.

Karena $x, y \in B(A)$, maka $(x \vee y) \vee(x \vee y)^{\sim}=(x \vee y) \vee 1=x \vee(y \vee 1)=$ $x \vee\left(y \vee y^{\sim}\right)=x \vee 1=x \vee x^{\sim}=1$. Oleh karena itu diperoleh bahwa $x \vee y \in B(A)$.

Perhatikan bahwa: $\beta(x \vee y)=\left(\beta_{a}(x \vee y), \beta_{a^{\sim}}(x \vee y)\right)=\left(a \wedge(x \vee y), a^{\sim} \wedge(x \vee y)\right)=$ $\left((a \wedge x) \vee(a \wedge y),\left(a^{\sim} \wedge x\right) \vee\left(a^{\sim} \wedge y\right)\right)=\left(a \wedge x, a^{\sim} \wedge y=(x, y)\right.$.

Jadi $\forall(x, y) \in M_{a}^{\prime} \times M_{a}^{\prime} \sim$ terdapat $x \vee y \in B(A)$ sehingga $\alpha(x \vee y)=(x, y)$, sehingga $\beta$ adalah epimorfisma.

Karena $\beta$ homomorfisma satu-satu dan pada, maka $\beta$ adalah isomorfisma, sehingga $B(A) \cong M_{a}^{\prime} \times M_{a^{\sim}}^{\prime}$.

\section{Kesimpulan}

Misal suatu sistem matematika $\left(A, \wedge, \vee,(\cdot)^{\sim}\right)$ adalah Pra $\mathrm{A}^{*}$-Aljabar. Selanjutnya diperoleh definisi dan sifat-sifat dari Pra $A^{*}$-Aljabar seperti pada bagian 2, serta diperoleh juga sifat-sifat dari $M_{x}$ seperti pada bagian 3. Dengan mendefinisikan $M_{a}^{\prime}=\{s \in B(A) \mid s \leq a\}$ dan $M_{a^{\sim}}^{\prime}=\left\{t \in B(A) \mid t \leq a^{\sim}\right\}$ dengan $B(A)=\left\{x \in A \mid x \vee x^{\sim}=1\right\}$ yang disebut senter (centre) dari $A$. Kemudian diperoleh Dekomposisi dari $A$ seperti pada bagian 4 .

\section{Ucapan Terima kasih}

Penulis mengucapkan terima kasih kepada Bapak Admi Nazra, Ibu Nova Noliza Bakar, Ibu Lyra Yulianti, Bapak Dodi Devianto, Bapak Zulakmal, yang telah 
memberikan masukan dan saran sehingga makalah ini dapat diselesaikan dengan baik.

\section{Daftar Pustaka}

[1] Satyanarayana, A. dan J.V. Rao. 2011. Representation of Pre A*-Algebra by Section of Sheaves. International Journal of Computational Cognition, Vol. 9, No. 2, June 2011 (40-44).

[2] Rao, J.V., S. Rao, dan D. Kalyani. 2011. Decomposition of Pre A*-Algebra. International Journal of Mathematical Sciences and Applications, Vol.1 (1) Januari 2011.

[3] Rao, J.V. dan S. Rao. 2009. Pre A*-Algebra as a Poset. African Journal of Mathematics and Computer Science Research, Vol 2 (4), May 2009 (073-080). 